\title{
A REVIEW OF THE AIR FORCE MATERIALS RESEARCH AND DEVELOPMENT PROGRAM
}

\author{
JUNIE J. BANKS
}

DONNA J. TATE

TECHNICAL DOCUMENTARY REPORT No. WADC-TR-53-373, SUPPL. 8

MARCH 1962

\author{
DIRECTORATE OF MATERIALS AND PROCESSES \\ AERONAUTICAL SYSTEMS DIVISION \\ AIR FORCE SYSTEMS COMMAND \\ WRIGHT-PATTERSON AIR FORCE BASE, OHIO
}

